The chapter 'Women, girls and alcohol' reviews evidence for 'convergence', or women's drinking catching up with that of men, and 'telescoping', or how in women the time from initiating drinking to dependence is shorter than in men. Prescription drug misuse, a growing problem in UK clinical practice, is also discussed. It is the one area of substance misuse where problems are as common in women as they are in men and it is frequently associated with dual diagnoses and chronic pain. Disappointingly, however, there is little on new treatment strategies and nothing at all on benzodiazepine misuse. The chapter dealing with pregnancy emphasises alcohol as the most important and most common teratogenic substance and it also discusses methadone as the treatment of choice in opiate users. The authors specifically state that the UK practice of detoxification from opiates in pregnancy is not supported by the evidence and is associated with high levels of relapse and complications of pregnancy.

The detailed section on biological issues in women explains the current state of knowledge on the neuroendocrine, genetic, pharmacokinetic and pharmacodynamic aspects of addiction as applied to women. This is rarely addressed in such detail in other texts and is probably the book's most useful section.

In general, the epidemiology applies to US populations and is therefore less relevant to UK readers. The chapter on ethnicity serves as a reminder of the importance of putting substance misuse into its historical, social, ethnic and cultural context but the detail is based on US ethnic groups. The legal chapter reviews the fascinating story of the approach to prosecution of drug-using pregnant women and compulsory drug testing in some US states and is more hopeful about the family drug courts model.

The book is a reminder of the importance of addressing the specific problems of women, but because of its US focus it does not help the UK reader with issues such as organising services.
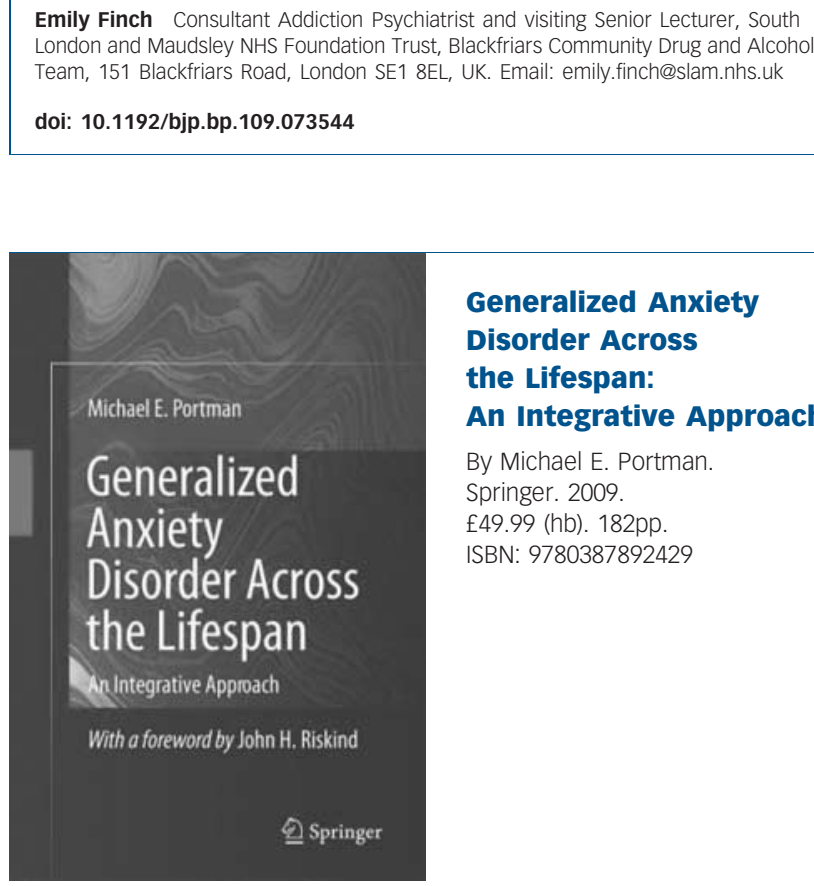

\section{Generalized Anxiety Disorder Across the Lifespan: An Integrative Approach}

By Michael E. Portman. Springer. 2009 £49.99 (hb). 182pp. ISBN: 9780387892429

After reading this book I thought more about the author than the subject. Michael Portman is a clinical social worker who both researches and treats generalised anxiety disorder (GAD). This background flavours the whole of this book. Portman almost certainly came across the subject as a genuine novice, but now is an avowed enthusiast of generalised anxiety disorder as a concept, a diagnosis and an important focus of treatment. I know the average reader does not get excited about GAD, but its unattractive acronym is appropriate in this case. 'By GAD', I can see Portman exclaim, 'what a diagnosis. It is like no other - and is mine to survey in all its glory'. So, rather like the revelation that Keats experienced when first looking at Chapman's translation of Homer, he feels 'like some watcher of the skies when a new planet swims into his ken', and he wants us to know all about it too.

By contrast I feel that GAD is better described as a 'god-awful diagnosis', as it is grossly heterogeneous, overlaps with almost every other psychiatric diagnosis known to man, and is singularly unhelpful in selecting treatment. So it might be expected that I would have a jaundiced view of this book. But I don't. It is actually quite refreshing to read unadulterated enthusiasm even when in my view it is slightly misplaced. We have a straight account of the symptoms, assessment, concepts and treatment of the disorder, not just in adults, but also in children and in older people, and by and large these are accurate, comprehensive and highly informative. There are also appendices that include seven scales for recording generalised anxiety disorder.

Although we gain by straight delivery of information, we sometimes lose in a lack of reflection and criticism. There are too many references to ex cathedra statements by 'international experts' and 'leading figures', but virtually no mention of the problems of dependence with benzodiazepines and other drugs, the influence of pharmaceutical companies on both prescribing and trial reporting, and the problems of management in primary care, where computerised cognitive-behavioural therapy is likely to be an important therapy source in the future. Still, this is an honest and useful book that is worth reading closely and which is an asset to the subject.

Peter Tyrer Centre for Mental Health, Imperial College London, London W6 8RP, UK. Email: p.tyrer@imperial.ac.uk

doi: 10.1192/bjp.bp.109.073494

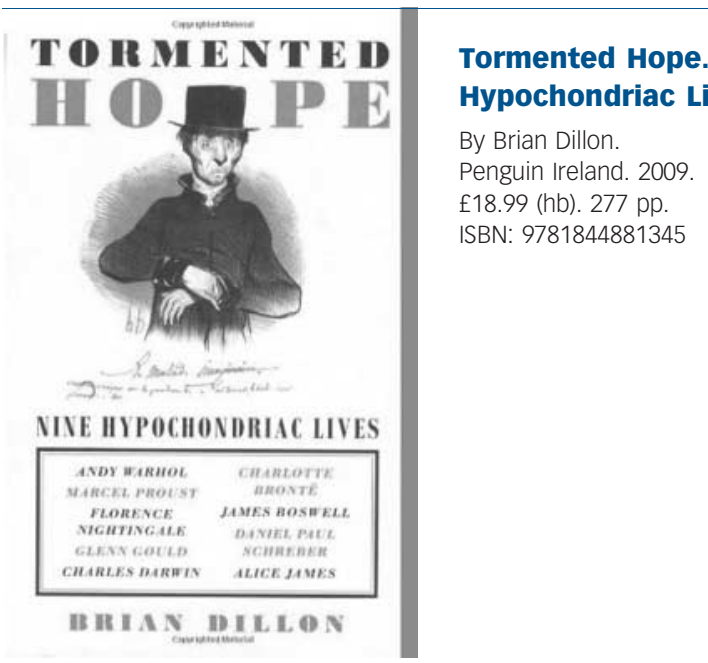

In this engaging book, the Irish writer Brian Dillon looks at the topic of hypochondria. The subject is an elusive one, because the concept of hypochondria has changed greatly over the centuries. The word hypochondrium was used by Hippocrates to refer to the regions of the abdomen under the ribcage. The Greeks believed that the underlying organs were the source of disordered emotions. Since then hypochondria has been deemed to be variously a 'nervous' disorder, a form of melancholia, a type of 